\title{
Turbulent Taylor vortex flow
}

\author{
By E. L. KOSCHMIEDER \\ College of Engineering and I. Prigogine Center for Statistical Mechanics, \\ The University of Texas, Austin
}

(Received 3 November 1978)

The wavelength of turbulent Taylor vortices at very high Taylor numbers up to $40000 T_{c}$ has been measured in long fluid columns with radius ratios $\eta=0.896$ and $\eta=0 \cdot 727$. Following slow acceleration procedures the wavelength (in units of the gap width) of turbulent axisymmetric vortices was found to be $\lambda=3 \cdot 4 \pm 0 \cdot 1$ with the small gap and about $\lambda=2 \cdot 4 \pm 0 \cdot 1$ with the larger gap, and thus in both cases substantially larger than the critical wavelength of laminar Taylor vortices. In the narrow and wide gap the wavelength was, within experimental error, independent of the Taylor number for $T>100 T_{c}$. In the experiments with the narrow gap a clear dependence of the value of the wavelength of the turbulent vortices on initial conditions was found. After sudden starts to Taylor numbers $>700 T_{c}$ the wavelength of steady axisymmetric turbulent vortices was only $2 \cdot 4 \pm 0 \cdot 05$, being then the same as the wavelength of the vortices after sudden starts in the wide gap, and being, within the experimental error, independent of the Taylor number. In the narrow gap all values of the wavelength between $\lambda_{\max }=3.4$ and $\lambda_{\min }=2.4$ can be realized as steady states through different acceleration procedures. In the wide gap the dependence of the wavelength on initial conditions is just within the then larger experimental uncertainty of the measurements.

\section{Introduction}

It has been known for a long time that Taylor vortices retain their basic toroidal structure discovered by G. I. Taylor (1923) also under turbulent conditions, that means at very high Taylor numbers. The oldest evidence pointing in this direction is the paper of Pai (1943) who studied turbulent flow between rotating cylinders. With regard to the type of flow between the cylinders Pai observed, 'It seems, therefore, that ring-shape vortices still exist at Reynolds numbers as high as several hundred times the critical Reynolds number'. The next study from which information about turbulent Taylor vortices can be obtained is the paper of Schultz-Grunow \& Hein (1956). They show a sequence of pictures demonstrating the onset of the Taylor instability, the transformation to doubly periodic flow, the transition to turbulence and finally axisymmetric turbulent vortices at around 200 times the critical Reynolds number. In Pai's and Schultz-Grunow \& Hein's studies it was observed that the size of the vortices increased with increased rotation rates. Pai noted a decrease of the number of vortices from 6 to 4 . Schultz-Grunow \& Hein found that the vortices at the highest rotation rates were 'about twice as long' as under critical conditions. Both observations are of a qualitative nature, Pai's apparatus was too short to permit small 
variations of the vortex size, and Schultz-Grunow \& Hein's paper is mainly a demonstration of a visualization technique.

The transition to turbulent Taylor vortex flow was first investigated systematically by Coles (1965). He studied the range of Taylor numbers from critical to about $100 T_{c}$, discovered the existence of doubly periodic flow and observed a dependence of the doubly periodic vortices on initial conditions. It seems that in his experiments the axial wavelength of the vortices increased on the average with increased Taylor numbers. Coles also presented photographs of turbulent vortices, but did not go into further detail other than in the case of spiral turbulence, which occurs when the cylinders counter-rotate. Spiral turbulence is discussed in detail in the paper of Van Atta (1966). A theoretical basis for the understanding of doubly periodic flow was then laid in a paper by Davey, DiPrima \& Stuart (1968). This investigation was continued by Eagles (1971) and Nakaya (1975); similar studies of the temporal development of Taylor vortices have been made by Yahata (1979).

The investigation of the transition to turbulence in Taylor vortex flow has been continued recently in experiments by Fenstermacher, Swinney \& Gollub (1979) and Walden \& Donnelly (1979). In these experiments detailed local measurements of the velocity of the flow at Taylor numbers ranging to a few hundred $T_{c}$ have been made. It was noted again that the toroidal structure of the flow is retained at even higher Taylor numbers under turbulent conditions. However, no further studies of turbulent vortices were made since the velocity distributions degenerate to broad bands of noise as turbulence is approached. It still remains to be determined precisely under what circumstances the size of the Taylor vortices increases and by how much if the doubly periodic regime is passed and the turbulent regime is entered. It is the purpose of the experiments to be described in the following to provide this information.

\section{Description of the apparatus}

The apparatus used is a slightly modified version of the apparatus described in detail in Burkhalter \& Koschmieder (1973), henceforth referred to as I, where a schematic section through the apparatus is shown. The outer glass cylinder was held stationary and was the same as that used in I, having an internal radius of $6 \cdot 285 \pm$ $0.006 \mathrm{~cm}$. Most experiments were made with a new inner brass cylinder with an outer radius of $5.633 \pm 0.005 \mathrm{~cm}$. The gap width is then $0.652 \pm 0.01 \mathrm{~cm}$ and the radius ratio $\eta=r_{i} / r_{0}=0 \cdot 896$. The larger of the two cylinders used in I with a radius of $4.572 \mathrm{~cm}$ and a gap of $1.713 \mathrm{~cm}$ and a radius ratio $\eta=0.727$ was also used in the present experiments. The fluid column was $80.5 \mathrm{~cm}$ long with the narrow gap and $84.9 \mathrm{~cm}$ long with the wide gap. There was then space for 122 critical vortices in the narrow gap and 50 vortices in the wide gap, making both columns reasonable approximations of the infinitely long column investigated in theoretical studies. A detailed investigation of the column length effects can be found in Cole (1976). The fluid columns had stationary end plates in all experiments. The temperature of the glass cylinder was held constant and at the temperature of the inner cylinder by water of $25^{\circ} \mathrm{C}$ circulated between the glass cylinder and a lucite cylinder surrounding the glass cylinder, and through the inner cylinder as well. This was done to eliminate any possible effect of radial temperature gradients. The fluid in the gap between the glass cylinder and the rotating inner cylinder was water. The fluid motions were made visible by aluminium powder 
suspended in the water. The vertical extension of the vortices were measured with a cathetometer accurate to $\pm 0.01 \mathrm{~cm}$.

The Taylor number in an apparatus with radius ratio $\eta$ is given by the formula (Roberts 1965)

$$
T=\frac{2 \eta^{2}}{1-\eta^{2}} \frac{\Omega^{2} d^{4}}{\nu^{2}}
$$

where $\Omega$ is the angular velocity, $d$ the gap width and $\nu$ the kinematic viscosity ( $\nu$ of water at $25^{\circ} \mathrm{C}=0.896 \times 10^{-2} \mathrm{~cm}^{2} \mathrm{~s}^{-1}$ ).

The wavelength is a non-dimensional measure of the size of the vortices, defined as the ratio of the vertical extension $2 l$ of two vortices divided by the gap width $d$. The theoretical significance of the wavelength is evident from the fact that the critical wavelength is one out of only two quantities which follow directly from linear stability analysis, the other quantity being the critical Taylor number. The wavelength measured in experiments is not necessarily the same as the wavelength determined theoretically for fluid columns of infinite length, because of end effects and the associated quantization condition. All wavelengths determined in the following experiments have been determined with the formula for the wavelength in a finite column, namely

$$
\lambda_{f i}=L / N d
$$

(see $\mathrm{I}$ ), where $L$ is the column length, $N$ is the number of vortex pairs (which are referred to as rings) and $d$ the gap width. Formula (2) does not make provision for end effects. It gives the average value of the wavelength in the entire column, regardless of whether the flow is doubly periodic or not. Since all measurements determined $\lambda_{f i}$ the subscript $f i$ will be dropped from now on.

\section{Wavelength measurements in the narrow gap}

The wavelength was determined as a function of the Taylor number using two methods to reach a given Taylor number, namely either the steady acceleration procedure or the sudden start procedure. It was observed in I and in Burkhalter \& Koschmieder (1974) (henceforth referred to as II) that the wavelength of laminar axisymmetric Taylor vortices is markedly different if a certain supercritical $T$ is reached either by a quasi-steady increase of the rotation rate or through a sudden start. The very high Taylor numbers studied in the present experiment do not permit the slow quasi-steady increase, rather the rotation rate had to be increased steadily. This is done by a motor which steadily turns the control of the motor turning the inner cylinder. But the increase of the rotation rate was made as slow as possible, namely at such a rate that the highest studied Taylor number was reached within $8 \mathrm{~h}$. The steady acceleration rate was then $7 \times 10^{-4} \mathrm{rad} \mathrm{s}^{-2}$. If experiments with lower Taylor numbers were made the same acceleration rate was used but the increase of $\Omega$ was then interrupted by time intervals in which the rotation rate was held constant. This procedure will be referred to as slow acceleration. In most experiments the average acceleration rate was substantially smaller than $7 \times 10^{-4} \mathrm{rad} \mathrm{s}^{-2}$. The sudden start experiments were conducted as described in II. The control of the drive motor was set for a rotation rate corresponding to a desired Taylor number while the motor was at rest. When the motor was switched on steady rotation of the inner cylinder 
was obtained within a second, which is a much shorter time interval than the viscous relaxation time $d^{2} / \nu$ of the fluid, which is of order of a minute.

Experiments in the moderately supercritical range up to about $6 T_{c}$ were made with a more viscous fluid, namely silicone oil of $0 \cdot 1 \mathrm{~cm}^{2} \mathrm{~s}^{-1}$ viscosity, because with water the rotation rate of the inner cylinder would have been too slow to obtain satisfactory results. Increasing the rotation rate very slowly from zero on, Taylor vortices were first observed to form at the top and bottom end plates. Details of the effects that the end boundaries can have on the Taylor vortices in a short column have been discussed by Benjamin (1978). Increasing $\Omega$ slowly caused the formation of additional vortices spreading from the ends until at $T_{c}$ the entire column was filled with a perfect pattern of laminar, axisymmetric vortices. The centre section of such a pattern is shown on figure 1 (plate 1). The measured critical wavelength $\lambda_{c}$ was then $\lambda_{c}=2 \cdot 13$, deviating by $+6 \%$ from the expected theoretical $\lambda_{c}$ which is 2.008 according to Roberts (1965). Note that the systematic uncertainty due to the quantization condition is $\Delta \lambda= \pm 0.03$. The uncertainty in the length of the column is of order of $0.1 \%$, the uncertainty of the gap width over the entire length of the column is of order of $\pm 1.5 \%$. That still leaves $3 \%$ experimental error unaccounted for, which must originate from variations of the size of the end rings, which are larger than the rings free from the ends as discussed in I. Increasing the Taylor number above critical caused transition to doubly periodic flow at $T=1 \cdot 26 T_{c}$. The axial wavelength remained unchanged until then.

Experiments with $T>5 T_{c}$ were made with water as the fluid. An example of doubly periodic flow at $7 T_{c}$ is shown on figure 2 (plate 2 ). Note that at this value of the Taylor number the wavelength has already increased by about $20 \%$ above $\lambda_{c}$. Under these conditions the flow is still perfectly laminar, without any time-dependent irregularities and can be maintained like that for hours. There is, however, non-uniqueness. Depending on the way in which a particular $T$ in this range has been realized, either very slowly or, say, through a sudden start, the axial and azimuthal wavelengths of various resulting steady states differ. This has been studied in detail by Coles (1965) and has therefore not been pursued here any further. Continuing the steady acceleration procedure, the axial wavelength of the flow increased, on the average, with increased $T$, and the azimuthal wavelength increased, on the average, as well. The qualifying phrase 'on the average' does not exclude that a particular state with a given axial and azimuthal wavelength at a given $T$ cannot be maintained if the Taylor number is varied. But if acceleration procedures are followed in which the fluid is never in true equilibrium and if the axial wavelengths of various experiments after different acceleration periods are compared, then it is found that the wavelength increases with $T$ on the average. The axial wavelength of the flow in several steady states, in which the rotation rate was held constant for at least an hour following a preceding steady acceleration procedure, are shown in figure 3 .

Proceeding with a steady acceleration up to $100 T_{c}$, the axial wavelength increased to a maximal value of about $\lambda_{\max } \simeq 3.4$. The average value of $\lambda_{\max }$ after ten steady acceleration experiments up to $100 T_{\mathrm{c}}$ is indicated in figure 3 by the open circle marked with a cross. With the increase of $T$ the flow became gradually irregular, so that at $100 T_{c}$ a truly laminar pattern did no longer exist. The transient irregularities of the flow persisted even if the rotation rate was held constant for hours at $100 T_{c}$. A picture of the flow in this state is shown on figure 4 (plate 3 ). Note that the azimuthal wavelength has increased also, there are now only two waves along the circumference, 


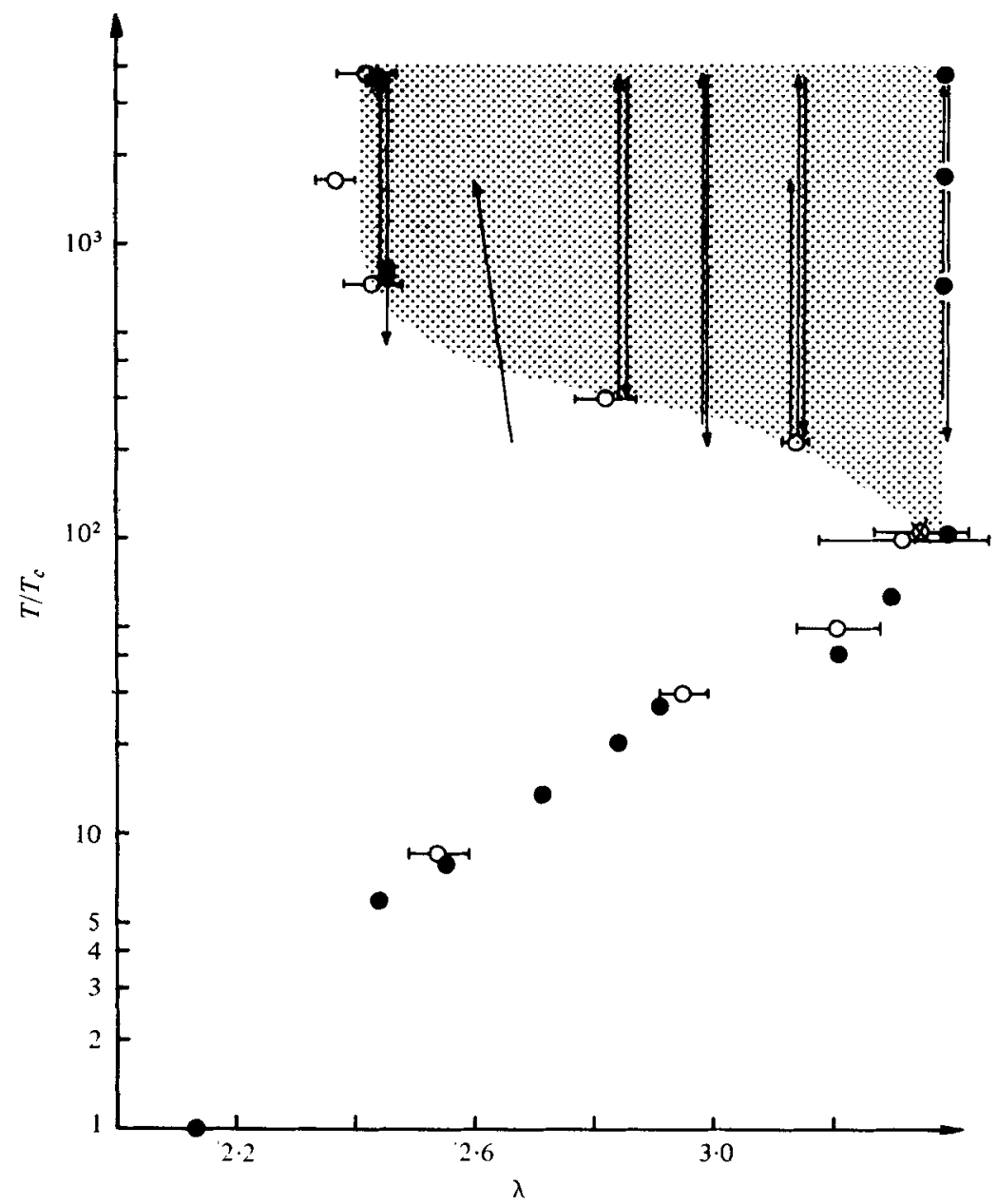

Figure 3. Wavelengths of Taylor vortices after steady acceleration experiments (๑) and after sudden starts (O) in the narrow gap. Arrows indicate shifts of the Taylor number. The shaded area indicates the region of non-uniqueness.

while there were six azimuthal waves on figure 2 . There were only two azimuthal waves at $100 T_{c}$ in each case that the azimuthal wavelength was determined.

A critical point seems to have been reached in this apparatus at around $100 T_{c}$. As we will see soon, the characteristics of the flow change markedly when $100 T_{c}$ is exceeded. We note in this context that in the velocity spectra of Fenstermacher et al. (1978) the broad band of noise which ultimately becomes turbulence appears at a Reynolds number of around $12 R e_{c}$, which corresponds to a Taylor number of $144 T_{c}$. The apparatus of Fenstermacher et al. and our apparatus differ, in particular in the ratio of the column length to gap width. Therefore one cannot expect precise agreement in the value of the Taylor number at around which turbulent Taylor vortex flow forms in both apparatus. Butit appears that, within the experimental uncertainty, the appearance of the broad band of noise in their experiments and the change of the characteristics of the flow in this experiment are compatible. In this experiment we 
say that at around $100 T_{c}$ the transformation to real turbulence begins. By real turbulence we mean that all three velocity components at any point in the fluid are randomly varying functions of time. The velocity components of the flow shown on figure 2 are certainly already functions of time at any point in the fluid; however the variations do not seem to be random yet. But the real justification for the statement that the transformation to turbulence begins at around $100 T_{c}$ in this experiment comes from the fact that from $100 T_{c}$ on the characteristics of the flow change drastically in order to match the behaviour of the flow under conditions when the flow is undoubtedly turbulent. That means (1) the wavelength of the vortices becomes independent of the Taylor number, while $\lambda$ definitely increased up to $100 T_{c}$; and (2) that the wavelength becomes again very clearly dependent on initial conditions, while at $100 T_{c}$ the wavelength was, within the experimental errors, see figure 3 , not dependent on initial conditions. We will now discuss these points in detail.

For $T>100 T_{c}$ the axial wavelength does not increase further but remains constant if the rotation rate is increased through steady acceleration. The two azimuthal waves that remained at $100 T_{c}$ gradually disappear and axisymmetric turbulent vortex flow is established at $T \simeq 700 T_{c}$. With axisymmetric turbulent vortex flow we mean axisymmetry in a statistical sense. This means in particular that the sinks of the vortices are aligned horizontally, as can be seen on figures 5 and 8 (plates 4 and 7 ). Under these circumstances there are, of course, also rapid small scale variations of the location of the sinks. As soon as the vortices are axisymmetric (in the mean) they become very stable with regard to either fast increases or fast decreases of the rotation rate of the inner cylinder, in a way strongly reminiscent of the behaviour of axisymmetric laminar Taylor vortices whose wavelengths remain also unchanged if the Taylor number is varied (over a large range), regardless whether that is done slowly or quickly, as was reported in II, figure 9 . For example, the wavelength $\lambda_{\max }$ of the turbulent vortices did not change if the Taylor number was increased in $30 \mathrm{~s}$ from $300 T_{c}$ to $3800 T_{c}$, or vice versa if the Taylor number was reduced from 3800 to $300 T_{c}$ in $30 \mathrm{~s}$. If the Taylor number is reduced one has to stay away from the doubly periodic nearly laminar regime in order to preserve the wavelength. Examples of such quick increases, respective decreases of the Taylor number, referred to as shifts, are indicated in figure 3 by the arrows. A photograph of steady turbulent axisymmetric flow with the maximal wavelength is shown on figure 5. Taylor numbers higher than $4000 T_{c}$ were not tried but there was no indication whatsoever that the behaviour of the flow was about to change.

Over the entire range of Taylor numbers the steady or slow acceleration experiments were supplemented with sudden start experiments. The sudden start experiments at each particular Taylor number were repeated at least 20 times in order to obtain a sufficient data basis for the determination of the standard deviation of the wavelength measurement. The standard deviations are indicated in figure 3 by the error bars. Note that there is also a systematic error associated with the wavelength measurements, caused by the quantization condition. Since we have stationary end plates at both column ends and since stationary end plates force the flow to form a sink at the location of the end plate (as discussed in $I$ ), both end conditions mean that variations of the average cell size smaller than $\pm l$ (where $l$ is the vertical extension of one vortex) do not change the number of cell pairs. Variations larger than $\pm l$ will either add or subtract a pair of cells from the column, which changes the wavelength according to 
formula (2). The standard deviations of all sudden start wavelength measurements were smaller, but not much, than the systematic error $\pm 1 / N$ of the wavelength measurement, except for the case at $100 T_{c}$, where the standard deviation was $\pm 1.50 / N$ which corresponds to an uncertainty of $\pm 4 \%$ of the wavelength.

The axial wavelengths of the vortices obtained by sudden starts in the range between 8 and $100 T_{c}$ are, within the experimental errors, the same as the wavelengths obtained by steady acceleration experiments, as is shown in figure 3 . In this apparatus with its long column, narrow gap, low viscosity fluid and the resting outer cylinder there is, in this range of Taylor numbers, only a modest degree of non-uniqueness, which is of about the order of the systematic uncertainty of the wavelength measurement.

Sudden starts to Taylor numbers $>100 T_{c}$ showed that the behaviour of the flow had changed significantly. As is shown in figure 3 the axial wavelength of the flow obtained after sudden starts is then much shorter than the wavelength of the flow after steady acceleration experiments. Doubly periodic turbulent vortices with two azimuthal waves formed after sudden starts to 211 and $300 T_{c^{*}}$ Figure 6 (plate 5) shows such doubly periodic turbulent vortices. At $T=211 T_{c}$ and $T=300 T_{c}$ the axial wavelength of the steady state is already clearly shorter than $\lambda_{\max }$. Note that at $211 T_{c}$ the standard deviation of the wavelength is very small $( \pm 0 \cdot 7 \%)$. This seems to be associated with the doubly periodic nature of the flow which apparently permits the vortices to adjust their wavelength even half an hour after the sudden start. That was similarly so at $300 T_{c}$. On the other hand, such a late adjustment of the wavelength never occurred with axisymmetric turbulent flow, where the pattern formation was always completed after $5 \mathrm{~min}$ at most. Increasing the Taylor number of the sudden starts to $700 T_{c}$ or more produced axisymmetric turbulent vortices with wavelengths of around $\lambda_{\mathrm{min}}=2.4$ which is substantially larger than the critical wavelength $\lambda_{c}$ of laminar flow. Within the error of measurement $\lambda_{\min }$ is independent of the Taylor number. Note that the range of Taylor numbers over which $\lambda_{\mathrm{m} 1 n}$ appears is very distorted in figure 3 by the semilogarithmic plot. The wavelength $\lambda_{\min }$ observed in the steady state after sudden starts is now about $30 \%$ smaller than the wavelength $\lambda_{\max }$ after steady acceleration experiments. Once formed after a sudden start the axisymmetric turbulent vortices retained their wavelength indefinitely and regardless of later increases or decreases of the Taylor number. For example the shift from 700 to $3800 T_{c}$ indicated in figure 3 did not change $\lambda$, although the shift was made in only $30 \mathrm{~s}$, that was as fast as the control of the motor could be turned by hand. It seems to be important to mention that the axisymmetric turbulent Taylor vortices form out of a seemingly chaotic motion (see figure 7 , plate 6 ) that arrives at the outer cylinder $0.5 \mathrm{~s}$ after the motor is switched on for a sudden start. A pattern of vortices is then established within a minute. It takes about 1 or at most 2 more minutes to smooth out differences in the size of individual vortex pairs (rings). The size distribution of all rings in the column will be discussed later. An example of axisymmetric turbulent vortex flow after a sudden start is shown on figure 8 (plate 7).

All wavelengths which are compatible with the formula $\lambda=L / N d$ and are in the interval between $\lambda_{\max }$ and $\lambda_{\min }$ can be realized as steady states (there are altogether 15 such states in this apparatus) at any Taylor number $>700 T_{c}$ by sudden starts to a Taylor number $100 T_{c}<T<700 T_{c}$ and a subsequent shift, as is indicated by the various arrows in figure 3 . The one arrow which does not point vertically is from a shift 


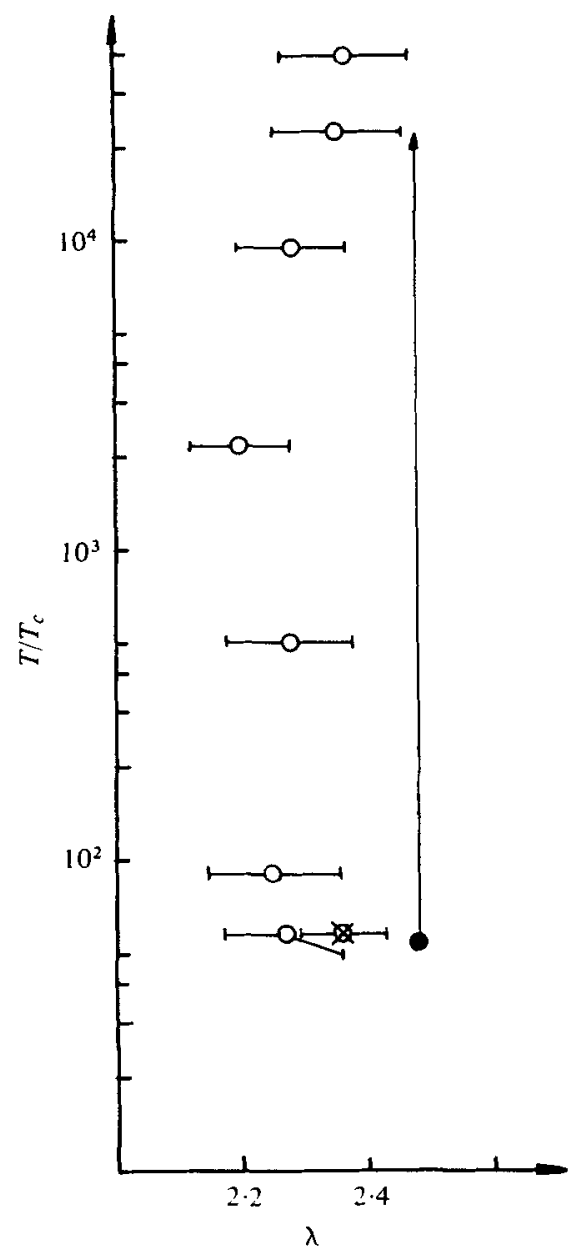

Figure 9. Wavelengths of Taylor vortices after slow acceleration experiments and after sudden starts $(O)$ in the wide gap. The arrow indicates a shift of the Taylor number.

made deliberately after a sudden start when the pattern formation was not yet completed. During the following change of the Taylor number the flow adjusted its wavelength through an increase of the number of vortices. This is, of course, not a reversible procedure. Reducing the Taylor number of the final state would not have changed the wavelength until the doubly periodic regime had been entered. If we extrapolate the observations with the various steady non-unique states at a given Taylor number $>700 T_{c}$ to the flow in an infinitely long cylinder then we find that there would be a continuum of possible steady states of turbulent vortices with all wavelengths ranging from $\lambda_{\max }$ to $\lambda_{\min }$. This is strikingly similar to the behaviour of laminar axisymmetric Taylor vortex flow where such a continuum of non-unique states exists likewise, as discussed in II, see in particular figure 12 therein. The nonuniqueness of the turbulent vortices can be seen easily with the naked eye and is demonstrated on figure 10 (plate 8).

We must finally ask whether with the development of turbulent flow the azimuthal 
periodicity, originally present in the azimuthal waves during the transition to turbulent flow, has disappeared completely. That does not seem to be the case. On figure 5 and in particular on figure 8 one can see that the interfaces between the different rings (the sinks) appear to be braided. Pursuing the motion visually one sees at the location of the sinks a distinct axial wave, travelling slowly in the direction of motion of the inner cylinder. The azimuthal wavelength of this wave appears to be fairly regular and is of order of the axial length of the vortices. It requires motion pictures to make this phenomenon easily visible.

We must finally show that $\lambda$, the average value of the wavelength of the flow, is indeed characteristic. We have to prove that the standard deviation of the wavelengths of all vortices measured individually is small as compared with the mean value of $\lambda$ and we have also to verify that the boundary conditions at the ends of the fluid column do not strongly affect the wavelength of all vortices. Table 1 gives samples of measurements of the vertical extension of turbulent vortex pairs (rings) normalized with the gap width, under various experimental conditions, together with the mean and the standard deviation of the wavelength of all individual rings. Also listed are the mean and standard deviation of the five top, as well as five centre and the five bottom rings and the mean and standard deviation of the rest of the rings (i.e. all rings except the five top and bottom rings) in the column, so that the effect of the top and bottom boundaries can be assessed. Of importance is the line with $\lambda$ (rest), which shows that the greatest part of the column around its centre has rings of very uniform size with standard deviations of $\pm 6 \%$ for 26 rings and $\pm 4 \%$ for 41 rings (at $1660 T_{c}$ as well as $3800 T_{c}$ ). The major contribution to these experimental uncertainties originates from the difficulty of pinpointing precisely the location of the sinks of the turbulent vortices. Nevertheless, the standard deviation of the wavelengths after steady acceleration (36 rings) is only $\pm 0.14 \mathrm{~cm}$, although in this case it appears to be hardly possible to locate the sinks with an accuracy of better than $\pm 0.1 \mathrm{~cm}$. For the sudden start experiments $(51$ rings) the standard deviation is $\pm 0.06 \mathrm{~cm}$, in this case the sinks are sharper. Note that the cathetometer accuracy is $\pm 0.01 \mathrm{~cm}$. In the rings free from the column ends there appears, however, to be a small gradual increase of the wavelength with increased depth along the column. This seems to be most apparent in the sudden start experiment with $3800 T_{c}$. The slope of the 41 rings of the centre was however only 0.002 , which makes it questionable whether the variation of the wavelength in the free part of the column is significant. In all cases discussed in table 1 there is clear evidence that the end rings are substantially larger than the average rings and, also, that the end effect carries over to the neighbouring rings at the bottom and to a lesser degree to the neighbouring rings at the top end. This is different from the observations reported in $I$, where, in laminar flow with resting end plates, the end rings were the only rings measurably affected by the boundary conditions.

\section{Wavelength measurements in a larger gap}

Since the wavelength of the turbulent vortices in the narrow gap depends so obviously on initial conditions we wondered whether this effect had been overlooked in the previous experiments described in $I$ and II, where the wavelength of turbulent flow in a gap with $\eta=0.727$ had already been measured. It was mentioned in I, figure $8(l)$, 


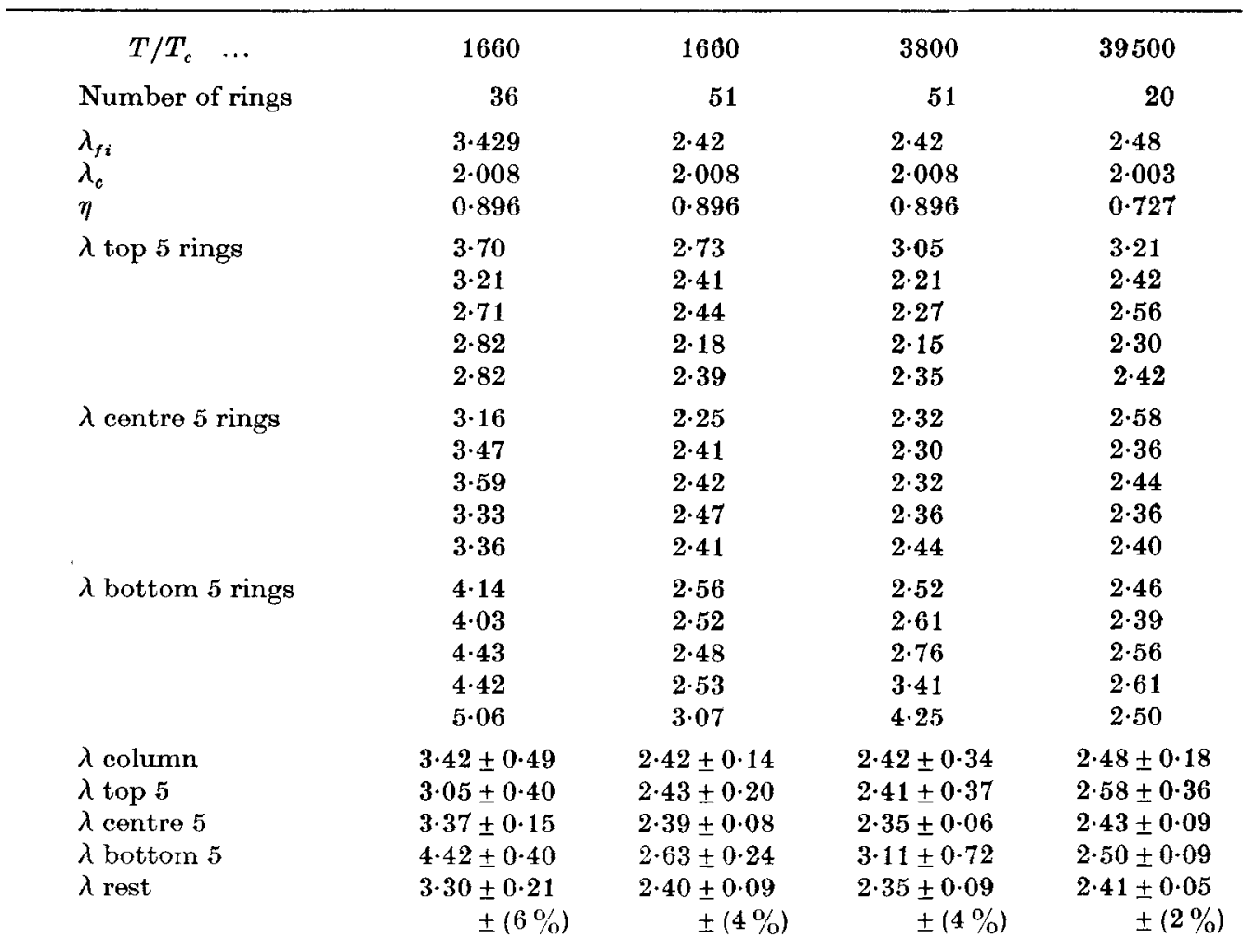

TaBLe 1. Wavelengths $2 l / d$ of individually measured Taylor vortices with the mean wavelength of the entire column and column sections, together with the standard deviations; in the narrcw and wide gap at different Taylor numbers.

that at $T=11300 T_{c}$ the wavelength was $\lambda=2 \cdot 54$, after a slow increase of the Taylor number. And in II, figure 10, it was shown that after sudden starts the wavelength ranged from $2 \cdot 2$ to $2 \cdot 4$ at $10000 T_{c}$. But no evidence for a dependence on initial conditions was noted. The experiments with the wide gap have therefore been repeated. The fluid used in these experiments was water, as in the case of the narrow gap. The results of sudden start experiments (at least 20 experiments at each point) and of slow acceleration experiments are plotted in figure 9. The open circle marked with a cross shows the average wavelength of ten slow acceleration experiments. When a wavelength was once established by this procedure, $\lambda$ did not change if the Taylor number was subsequently increased slowly or quickly to the highest Taylor number tried $T=40000 T_{c}$. The wavelengths obtained after sudden starts are smaller but are, within the experimental error, compatible with the average wavelength of slow acceleration experiments. The wavelength after sudden start experiments in the wide gap coincide, within the experimental error, with the wavelength observed after sudden start experiments in the narrow gap, when the Taylor number is greater than $1000 T_{c}$. Note that the absolute value of the experimental errors on figure 9 is larger than on figure 3 , because of the reduced number of vortex pairs in the column. The standard deviation of the wavelength after sudden start experiments with the large gap was about $0 \cdot 9$ times \pm 1 ring, which is very close to the systematic error of 
the wavelength measurement. Since, with the wide gap, there are usually only 22 rings in the column, the systematic uncertainty of $\lambda$ is consequently fairly large $(\sim 5 \%)$. The uncertainty of the wavelength of all vortices in the wide gap has been measured and is shown in the last column of table 1. With the wide gap there is, as figure 9 shows, no clear-cut dependence of the wavelength on the initial conditions, though the wavelength after sudden starts was on the average shorter than the wavelength after a slow acceleration at the same Taylor number. The failure to find an unambiguous consequence of the initial conditions seems to be due to two reasons. (1) Doubly periodic flow in the wide gap never develops many azimuthal waves, which seems to be necessary for a substantial increase of the axial wavelength. Consequently the difference in axial wavelengths after sudden starts and quasi-steady experiments must be smaller with a wide gap. (2) The resolution of the measurements (which is $\pm \lambda$ ) decreases with increased gap width (if the column length is the same). Smaller effects of initial conditions can therefore not be identified clearly.

We have finally checked whether a finite azimuthal disturbance has a significant effect on turbulent Taylor vortices. As in a previous experiment with laminar axisymmetric Taylor vortex flow (Koschmieder 1975), an oval brass bar $1.25 \mathrm{~cm}$ wide at its bottom and $0.3 \mathrm{~cm}$ deep at its apex (corresponding to $17 \%$ of the gap width) was attached to the inside of the glass cylinder over the entire length of the column. As in the laminar case such a disturbance causes a wake to form behind the brass bar which extends in the turbulent flow not further than one times the width of the bar. There is, within the experimental error, no noticeable effect of the disturbance on the wavelength, either after slow acceleration or after sudden starts. In other words, the turbulent vortices are stable against such a finite azimuthal disturbance.

\section{Discussion}

The experiments described above have shown that the flow of a fluid in the gap between a rotating inner and stationary outer vertical cylinder at very high Taylor numbers is well organized in the form of toroidal turbulent Taylor vortices of very uniform size. It was found that the wavelength of the turbulent vortices is substantially larger than the critical wavelength of laminar Taylor vortices. Taken together with earlier observations of Pai (1943) and Schultz-Grunow \& Hein (1956) and the observations in I and II, the present observations seem to make it certain that the wavelength of turbulent vortices is indeed larger than $\lambda_{c}$. We believe this to be a significant fact. It is known from experimental observations that the wavelength of convective motions caused by heating from below (the Bénard instability) increases with increased supercritical Rayleigh number. Considered together the increase of the wavelength in Bénard convection and Taylor vortex flow may express a more general feature of such instabilities. A discussion of a possible qualitative explanation of the increase of the wavelength has been given elsewhere (Koschmieder 1978).

A startling discovery of the experiments described above was the clear-cut dependence of the wavelength of the turbulent vortices on initial conditions as well as the surprising functional similarities in the behaviour of laminar axisymmetric Taylor vortices and turbulent axisymmetric Taylor vortices; namely the independence of the wavelength of axisymmetric vortices from the Taylor number and the existence of a continuum of steady non-unique states in both cases. We are not aware of a 
theoretical explanation for these effects. To the best of our knowledge a theoretical analysis of turbulent Taylor vortex flow has not yet been made. The very recent review of nonlinear hydrodynamic stability, in particular of Taylor vortex flow, by DiPrima (1978) does not mention such a study either. We believe, however, that the above experiments made with a simple apparatus under unambiguous conditions using a conventional fluid provide good material to test theories of axisymmetric turbulence, which go back to the studies of Batchelor (1946) and have been pursued in recent years in particular with numerical methods, e.g. Lilly (1971) and Herring et al. (1974).

We are, of course, aware of the great difficulties of a theoretical description of turbulent Taylor vortex flow. The difficulties originate from the truly nonlinear nature of the problem and will be enhanced if time-dependent initial conditions are to be considered. We believe that the wavelength, i.e. the size of the vortices, is of prime importance in the understanding of the problem. The velocity distribution for example depends obviously on the wavelength of the flow. The essence of the experimental results presented in our study can, in principle, also be obtained by local velocity measurements, although that would be extraordinarily laborious. It will furthermore become increasingly difficult to extract meaningful data out of the rapidly increasing noise in the velocity distributions.

A referee has pointed out to us that Dr F. R. Mobbs has likewise made an experimental study of Taylor vortices at very high Taylor numbers. His experiments show also the existence of axisymmetric turbulent Taylor vortices at Taylor numbers up to $80000 T_{c}$. The results of this investigation are presented in the paper by Barcilon et al. (1979). The axial wavelength of the flow and the variations of $\lambda$ are not discussed in this paper.

Financial support of the Bureau of Engineering Research to buy and machine the new inner cylinder of this apparatus is gratefully acknowledged.

\section{REFERENCES}

Barciton, A., Brindley, J., Lessen, M. \& Mobbs, F. R. 1979 (To be published.)

Batchelor, G. K. 1946 Proc. Roy. Soc. A 186, 480.

Benjamin, T. B. 1978 Proc. Roy. Soc. A 359, 27.

Burkhalter, J. E. \& Koschmieder, E. L. 1973 J. Fluid Mech. 58, 547.

Burkhalter, J. E. \& Koschmieder, E. L. 1974 Phys. Fluids 17, 1929.

CoLe, J. A. 1976 J. Fluid Mech. 75, 1.

COLES, D. 1965 J. Fluid Mech. 21, 385.

Davey, A., DiPrima, R. C. \& Stuart, J. T. 1968 J. Fluid Mech.31, 17.

DiPrima, R. C. 1978 Eighth U.S. Nat. Congr. Appl. Mech., UCLA.

EAGLES, P. M. $1971 J$. Fluid Mech. 49, 529.

Fenstermacher, P. R., Swinney, H. L. \& Gollub, J. P. 1979 J. Fluid Mech. (to appear).

Herring, J. R., Orszad, S. A., Kraichnan, R. H. \& Fox, D. G. 1974 J. Fluid Mech. 66, 417.

Koschmieder, E. L. 1975 Phys. Fluids 18, 499.

Koschmteder, E. L. 1978 Proc. XVII Int. Solvay Congr. Phys. (to appear).

LILLY, D. K. 1971 J. Fluid Mech. 45, 395.

NAKaYa, C. 1975 J. Phys. Soc. Japan 38, 576.

Pat, S. I. 1943 N.A.C.A. Tech. Note no. 892. 
Roberts, P. H. 1965 Proc. Roy. Soc. A 283, 550.

Schultz-Grunow, F. \& Hern, H. 1956 Z. Flugwiss. 4, 28.

TAYlor, G. I. 1923 Phil. Trans. Roy. Soc. A 223, 289.

VAN ATTA, C. $1966 J$. Fluid Mech. 25, 495.

Walden, R. W. \& Donnelly, R. J. 1979 Phys. Rev. Lett. 42, 301.

Yahata, H. 1979 Prog. Theor. Phys. (to appear). 